\title{
Editorial: Special Issue on Cognitive Radio Networks and Communications
}

\author{
Yan Zhang • David Grace • Lingyang Song
}

Published online: 23 April 2010

C) Springer Science+Business Media, LLC. 2010

Cognitive radio networks and communications is an emergent paradigm to address the spectrum scarcity challenge in the wireless community. Currently, different wireless systems are regulated by a fixed spectrum assignment strategy. This policy partitions the whole spectrum into a large number of different ranges. Each piece is exclusively used by a specific wireless system. This leads to an undesirable situation that some systems may only use the allocated spectrum to a very limited extent while others have very serious spectrum insufficiency situation. Cognitive radio is believed to be a highly potential technology to address these issues.

However, it is still in the very early stage of research and development. There are a number of technical, economical, and regulatory challenges to be addressed. This special issue aims to foster the dissemination of high quality research in cognitive radio networks and communications. Original papers are solicited in all aspects of cognitive radio including physical layer issues, architectures, protocol designs, theoretical studies, practical applications, and experimental prototypes.

To guarantee the quality, in this special issue, we only include the following eight papers at this special issue.

The first paper, entitled "Evaluation of Dynamic Channel and Power Assignment for Cognitive Networks", introduces a unifying optimization formulation to describe the dynamic channel and power assignment (DCPA) problem and an evaluation method for comparing DCPA algorithms.

The second paper is entitled "Joint Bandwidth and Power Allocations for Cognitive Radio Networks with Imperfect Spectrum Sensing". In this paper the authors study the joint

Y. Zhang

Simula Research Laboratory and University of Oslo, Oslo, Norway

D. Grace

University of York, York, UK

L. Song $(\varangle)$

Department of Electronics Engineering and Computer Science, Peking University, 100871 Beijing, China e-mail: lingyang.song@pku.edu.cn 
bandwidth and power allocations for Cognitive Radio Networks, which opportunistically operate on a set of channels unused by multiple Primary User (PU) Networks. An efficient joint allocation algorithm is proposed to solve the interference minimization problem with imperfect spectrum sensing.

The third paper, "Economic Approaches for Cognitive Radio Networks: A Survey", presents an extensive summary of the related work that use economic approaches such as game theory and/or price theory/market theory to model the behavior of primary and secondary users for spectrum sharing and discuss the associated issues.

The fourth paper is entitled "Analysis of QoS Provisioning in Cognitive Radio Networks: A Case Study". In this paper, the authors present a case study of key dynamic spectrum Access (DSA) protocol characteristics necessary for QoS provisioning. Specifically, personal/portable CR system that supports high quality multimedia (including HDTV) streaming over UHF frequency bands is chosen to consider. The results show the effectiveness of the proposed DSA QoS provisioning approach in sustaining high levels of QoS, e.g., supporting HDTV streaming in TV bands. This outcome is significant as FCC has recently approved UHF bands for unlicensed operations in the USA, and various DSA-based CR systems are being actively designed by the wireless industry.

The fifth paper is entitled "Cognitive Radio with Reinforcement Learning Applied to Multicast Downlink Transmission with Power Adjustment”. This paper shows how channel assignment in multicast terrestrial communication systems with distributed channel occupancy detection can be improved using intelligence based on reinforcement learning and transmitter power adjustment. The paper also shows how a power adjustment technique is developed which significantly reduces the level of overlap between adjacent base stations, and further reduces interference and transmitter power.

The sixth paper, "Reinforcement Learning Enhanced Iterative Power Allocation in Stochastic Cognitive Wireless Mesh Networks", presents an intelligent policy based on reinforcement learning to acquire the stochastic behavior of primary users. Based on the learning approach, the secondary users can adapt to the dynamics of the interference environment state and reach new NEs quickly through partially cooperative information sharing via a common control channel.

The seventh paper, "Satellite-Based Spectrum Sensing for Dynamic Spectrum Sharing in Ground-Located CRNs", presents a novel satellite-based spectrum sensing method for cognitive radio networks using multibeam antennas in large-scale coverage ground areas. This approach overcomes the limitations of the traditional sensing method, which therefore considerably improves the spectrum utilization. In addition, the proposed sensing method provides a new vision on the large-scale dynamic spectrum sharing system design to effectively integrate the terrestrial

The eighth paper, entitled "A Distributed Message-passing Approach for Clustering Cognitive Radio Networks", introduces a novel approach to form efficient node clusters in an ad hoc cognitive radio network based on the affinity propagation (AP) message-passing technique. With this approach, nodes exchange messages containing local network information with their direct neighbors until a high quality set of clusterheads and an efficient cluster structure emerges. The groupings are based on measures of similarity between the network nodes, which are selected based on application requirements.

In closing, we would like to thank all of the authors who have submitted papers to this Special Issue. We thank all anonymous reviewers who spent much of their precious time reviewing all the papers. Their timely reviews and comments greatly helped us select the best papers in this special issue. Finally, we hope you will enjoy reading the great selection of papers in this issue. 


\section{Author Biographies}
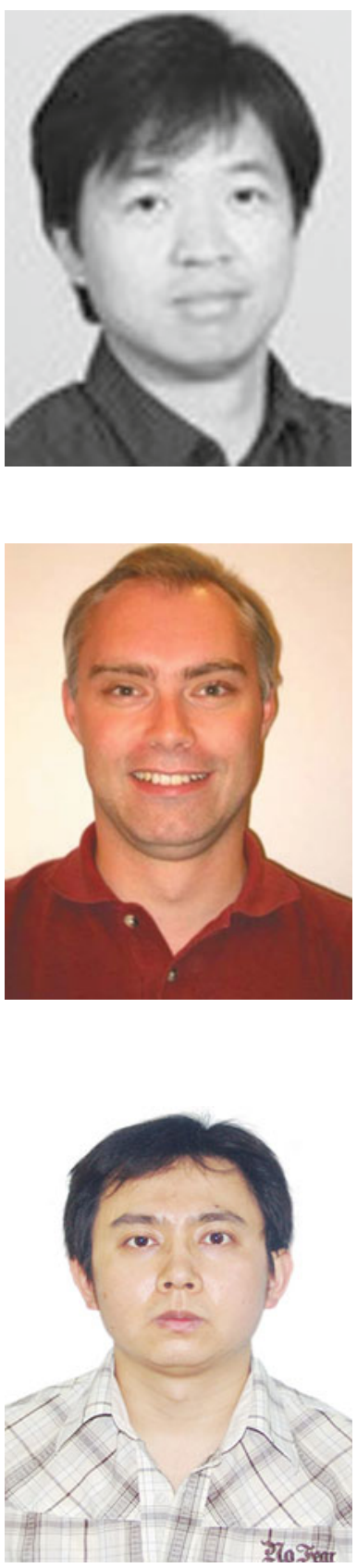

Yan Zhang received his Ph.D. from School of Electrical \& Electronics Engineering, Nanyang Technological University, Singapore. From August 2006, he has been working with Simula Research Laboratory, Norway. He is a regional editor, associate editor or on the editorial board of many international journals. He is currently also the Book Series Editor for the book series on Wireless Networks and Mobile Communications (Auerbach Publications, CRC Press, Taylor \& Francis Group) and is serving as co-editor for several books. He has been the organizing committee chair for many international conferences and is a member of Technical Program Committee for numerous international conferences. His research interests include resource, mobility, spectrum, data, energy and security management in wireless networks and mobile computing. He is a member of IEEE and IEEE ComSoc.

David Grace is Head of Communications Research Group and Senior Research Fellow within the Department of Electronics at the University of York. Current research interests include cognitive radio, particularly applying distributed artificial intelligence to spectrum assignment, and cognitive networking, interference management and coexistence. Currently, he is a co-investigator of the FP7 BuNGee project dealing with broadband next generation access, and recently he was the principal investigator of a UK MOD project on 'Cognitive Routing for Tactical Ad Hoc Networks'. From 2003-2007 he was the technical lead for the 14-partner FP6 CAPANINA project. He is currently chair of the Worldwide Universities Network Cognitive Communications Consortium, which has $50+$ member organisations worldwide. He is an author of 140+ papers, and a member of COST IC0902 and the IEEE Committee on Cognitive Networks. From 2005-2009 he was COST 297 WG1 chair which dealt with radio communications for high altitude platforms. In 2000, he jointly founded SkyLARC Technologies Ltd, and was one of its directors.

Lingyang Song received the B.S. degree in communication engineering from Jilin University, China in 2002 and a Ph.D. in differential space time codes and MIMO from the University of York, UK, in 2007, where he received the K. M. Stott Prize for excellent research. From January to September 2003 he worked as a software engineer in Hwasun Tomorrow Technology, Beijing. He worked as a postdoctoral research fellow at the University of Oslo, Norway, until rejoining Philips Research UK in March 2008. Now, he is with School of Electronics Engineering and Computer Science, Peking University, China. $\mathrm{He}$ is co-inventor of a number of patents and author or co-author of over 80 journal and conference papers. He is currently on the Editorial Board of International Journal of Communications, Network and System Sciences, Journal of Network and Computer Applications, and International Journal of Smart Homes, and a guest editor of Elsevier Computer Communications and EURASIP Journal on Wireless Communications and Networking. He serves as a member of Technical Program Committee and Co-chair for a number of international conferences and workshops. He is a member of the IEEE and IEEE ComSoc. 